\title{
Research on Goods and the Ship Interaction Based on ADAMS
}

\author{
Fangzhen Song , Yanshi He and Haining Liu
}

School of Mechanical Engineering, University of Jinan, Jinan, 250022 , China

\begin{abstract}
The equivalent method of the relative movement goods on board is discussed in details. This method is to establish dynamic model based on moving trajectory of gravity-center for goods and to take rigid body geometric model with the trajectory as constraints in ADAMS. The difference of simulation methods for the different goods in carrier rolling is compared. The interact of relative moving objects with bulk carrier is discussed by using the ADAMS model. It is verified that the ballast water can maintain the ship's stability by means of the ADAMS model.
\end{abstract}

\section{Introduction}

Ships often encounter all sorts of external force such as wind at anchor or voyage, so the equilibrium state of ships would destroy. Ships under external torque will be imbalanced. At this time, if the ship has proper stability, the external torque will be offset by the restoring moment which are the joint action of buoyancy and its own gravity, in order to avoid the tilt of ship continuing to expand[1]. When the external torque is eliminated, the restoring moment makes the ship (after a certain cyclical swing) to return to the original balance position. This kind of restoring ability of ship is the stability [2] problem of the ship that we study. The resilience of the ship is the problem of ship's stability that we are studying on.

The relative movement of goods on a ship can lead to the instability of the ship [3], [4]. When the ship leaves the equilibrium position and swing occurs, they intensify the oscillation amplitude of the ship and cause capsizing. It is necessary to consider relative movement of goods as the instability factors. The ship's stability is usually improved by setting ballast water tank on the ship [5], The ballast water of ballast cabin will help reduce the ship's sloshing caused by the swaying of goods. When we study the stability of ship in ADAMS[6], in order to simulate a more realistic motion of the ship and get more accurate results of the analysis, we must consider the effect of the swaying of goods and ballast water.

In this paper, grain is taken as an example of the relative movement of the goods. When grain is in natural grain piles, the nature of the flow in all directions into a cone is called grain scattered. The property of grain scattered is usually expressed as repose angle [7]. Repose angle is the angle between the cone slope that grain naturally forms falling from high and the bottom horizontal line. Repose angle is inversely proportional to scattered property. Good scattered property with small repose angle and poor scattered property with big repose angle[8].

\section{The equivalent of goods}

The repose angle of grains is $20^{\circ}$. It means when the stacked grain's surface is flat and the angle of container is more than $20^{\circ}$, grains begin to flow.

The trajectory's change of center of gravity is calculated when the cargo lateral tilt changes from $20^{\circ}$ to $40^{\circ}$. The calculation model of goods is built in Solidworks. It is shown in Fig. 1.

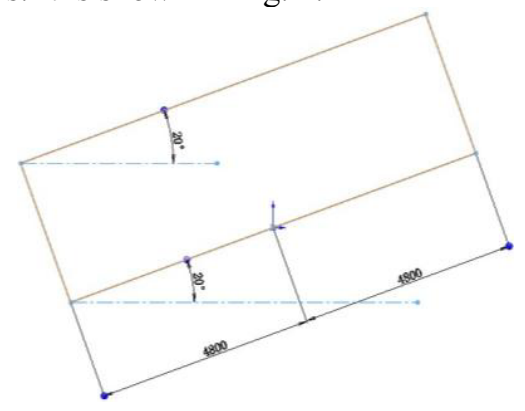

Figure 1. Calculation model of goods.

Bottom edge is $\mathrm{h}$ and the two lines are perpendicular to the bottom: the left line is a, the right line is $b$. When bottom edge is horizontal, two lines are perpendicular to the bottom are $c$. Cargo volume does not change, so $h \times c=h / 2 \times(a+b)$, then $2 c=a+b$.It is known that $h=9600 \mathrm{~mm}$. An angle between surface and the bottom of the cargo goods is set as $\theta . c=2750 \mathrm{~mm}$, so $2 a-9600 \tan \theta=5500$.

Every time change the angle of $2^{\circ}$, so the values of 10 times's change are 2917.6197, 3085.6487, 3254.5003, $3424.5960,3596.3695,3770.2715,3946.7744,4126.3779$, 
4309.6145, 4497.0571.

Get the change of barycentric coordinates as shown in Table 1:

Table 1. Barycentric coordinates

\begin{tabular}{|c|c|c|c|c|c|}
\hline $\begin{array}{c}\mathrm{Nu} \\
(\mathrm{m})\end{array}$ & $\mathrm{x}$ & $\mathrm{y}$ & $\begin{array}{c}\mathrm{Nu} \\
(\mathrm{m})\end{array}$ & $\mathrm{x}$ & $\mathrm{y}$ \\
\hline 1 & -606.145 & 1239.923 & 6 & -1265.483 & 905.001 \\
\hline 2 & -740.443 & 1182.932 & 7 & -1394.694 & 822.522 \\
\hline 3 & -873.343 & 1121.032 & 8 & -1523.552 & 734.585 \\
\hline 4 & -1005.022 & 1054.141 & 9 & -1652.345 & 641.023 \\
\hline 5 & -1135.667 & 982.167 & 10 & -1781.398 & 541.642 \\
\hline
\end{tabular}

Draw a line with the data. It is shown in figure 2:

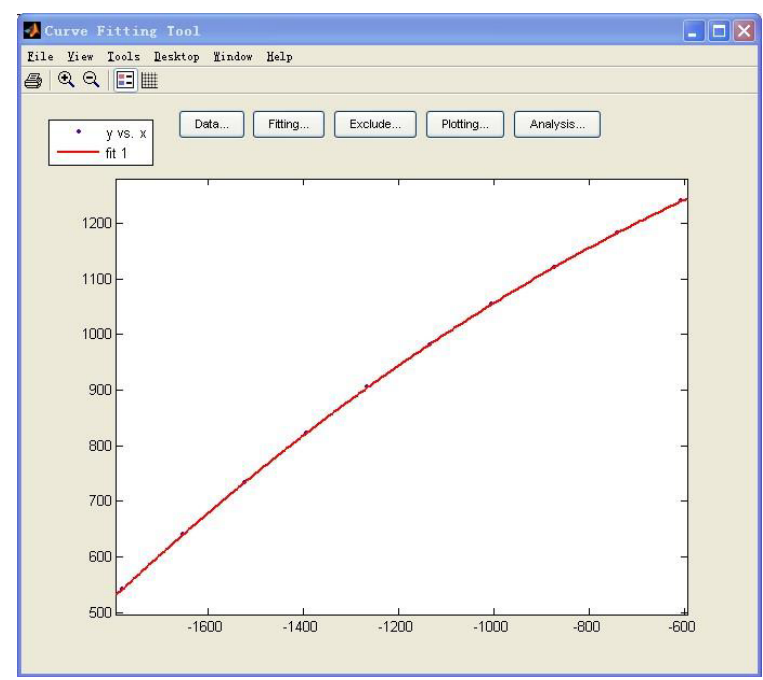

Figure 2. Barycentric coordinates curve.

Follow-up coordinate system is established. $x_{1}$ axis is horizontal to bottom line, $y_{1}$ axis is vertical to bottom line and the origin is coincidence with the one of the original coordinate system. Set the lines (attached by the point of measurement and the origin point) and $\mathrm{x}$ axis's angle is acute angle, the conversion formula is below:

$$
\left\{\begin{array}{l}
\cos (\theta+\alpha) \sqrt{x^{2}+y^{2}}=-x_{1} \\
\sin (\theta+\alpha) \sqrt{x^{2}+y^{2}}=y_{1}
\end{array}\right.
$$

The transformed coordinates are in table 2

Table 2. barycentric coordinates.

\begin{tabular}{|c|c|c|c|c|}
\hline Num & $\alpha$ & $\theta+\alpha$ & $x_{1}$ & $y_{1}$ \\
\hline 1 & 63.948 & -41.948 & -97.52406 & 1376.703 \\
\hline 2 & 57.956 & -33.956 & -195.2855 & 1381.828 \\
\hline 3 & 52.080 & -26.08 & -293.516 & 1390.428 \\
\hline 4 & 46.366 & -18.366 & -392.504 & 1402.577 \\
\hline 5 & 40.854 & -10.854 & -492.4445 & 1418.411 \\
\hline 6 & 35.570 & -3.57 & -593.6175 & 1438.086 \\
\hline 7 & 30.530 & 3.47 & -696.3057 & 1461.804 \\
\hline
\end{tabular}

\begin{tabular}{|c|c|c|c|c|}
\hline 8 & 25.741 & 10.259 & -800.8058 & 1489.811 \\
\hline 9 & 21.204 & 16.796 & -907.4028 & 1522.424 \\
\hline 10 & 16.912 & 23.088 & -1016.473 & 1559.98 \\
\hline
\end{tabular}

Draw a line with the data. It is shown in Fig. 3.

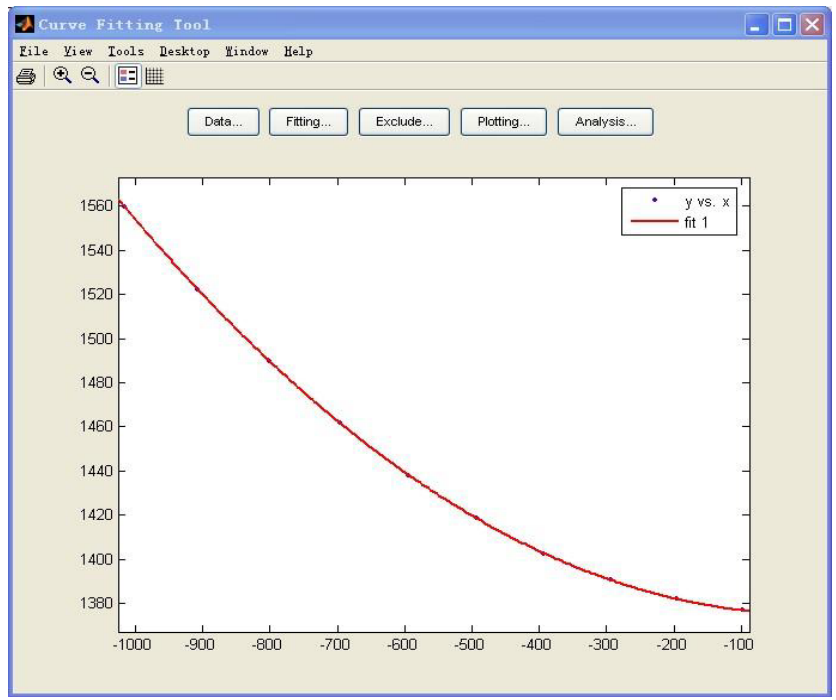

Figure 3. Barycentric coordinates curve.

The rigid body under the constraint of barycentre trajectory is shown in Fig. 4.

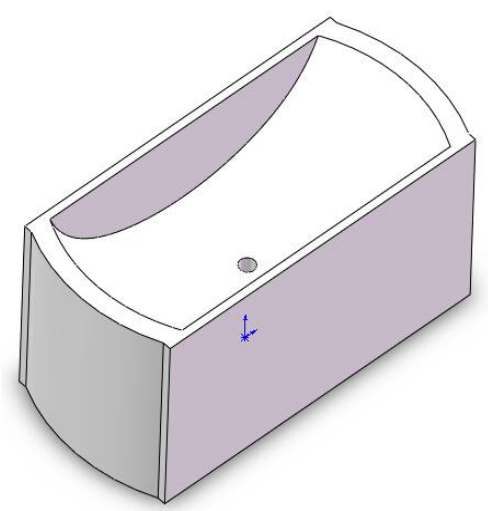

Figure 4. Rigid body under the constraint of barycentre trajectory.

The medial surface is drawn according to the calculated curve. The size of the hole which is in the middle of surface matches the spherical rigid body which is the simulation of center of gravity. When the angle of rigid body reaches $20^{\circ}$, center of gravity of the spherical rigid body is just through the hole on the edge in the vertical direction. It means that when angle exceeds $20^{\circ}$, hole edge can no longer constraint of the spherical rigid body, the spherical rigid body begin to roll along the inside surface of the rigid body. Establish contact constraint between rigid body and spherical rigid body. The quality of the rigid body is set as a small value which affect can be ignored. The quality of spherical rigid is set as the original quality of the goods after divided into 
segments. Damping is associated with speed. In order to prevent the spherical rigid body due to the inertia caused reciprocating rolling, so set a larger contact constraint damping coefficient to control the velocity of the spherical rigid body.

\section{The simulation of the ship in the water}

In this paper, we choose a $54 \mathrm{~m}$ motor cargo ship as the research object. In order to get a more realistic simulation of ship motion in the water, the spring is used as simulation of water surface support. Due to the spring is stretch spring, it cannot provide constraints when the force is perpendicular to the direction of the spring. The ship model will dump and then affect the simulation. The spring which is added to the bottom of the boat is changed to be added to the top of the boat to make hull be on suspension state. No matter how the hull is swinging, the hull can be restored to a state of balance due to gravity. This process has no effect on the size of force, only change the force's direction.

In order to avoid the position of gravity center and the suspension point of each section of the hull affecting the balance state of the ship, applying the spring at the four corners of the rigid deck surface of every section to ensure each rigid deck is level.

For spring stiffness, the hull support stiffness of the overall water is gotten according to the contact water area of the hull bottom, and then be divided equally to each of the spring unit of the hull support.

$F=\rho V \mathrm{~g}$, and $F=n k x, \rho S x \mathrm{~g}=n k x, \rho S \mathrm{~g}=n k$, so $k$ $=\rho S g / n$.

$\rho$ is the density of leaching liquid, $V$ is the volume of leaching liquid, $\mathrm{g}$ is acceleration of gravity, $n$ is the number of spring, $k$ is spring stiffness, $x$ is spring elongation.

In this paper, $n=78, \rho=1300 \mathrm{~kg} / \mathrm{m}^{3}, \mathrm{~g}=9.8 \mathrm{~N} / \mathrm{kg}$, $S=397.1117 \mathrm{~m}^{2}$,

So each spring stiffness is $\mathrm{k}=\rho \mathrm{Sg} / \mathrm{n}=1300 \times 397.1117 \times 9.8 / 78=64861.5777 \mathrm{~N} / \mathrm{m}$

The model is established in the ADAMS is shown in Fig. 5.

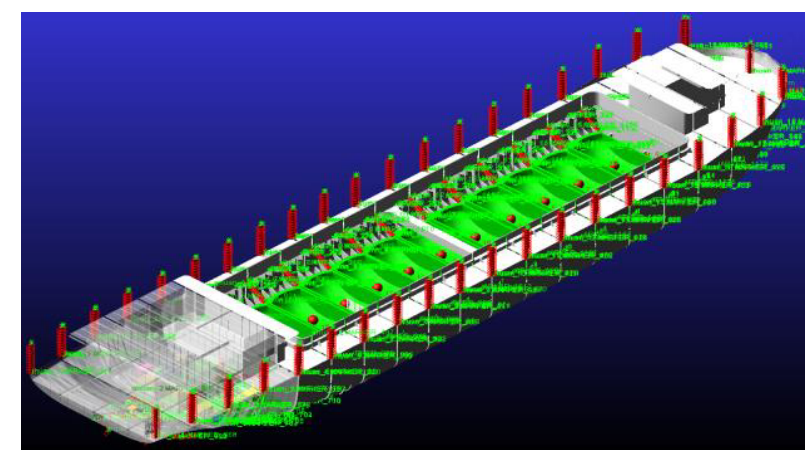

Figure 5. The model in ADAMS.

\section{Influence between relative moving objects and bulk carrier}

Two kinds simulation way of goods are established. The model with rigid block simulation is called model 1 and the model in the center of gravity trajectory simulation called model 2. First of all, the difference of two models in kinematics is compared. Under the action of the wind and waves, the ship will leave the original balance position to one side, and under the action of the inertia, the ship will reciprocating motion. The ship with bow and stern vertical line with the line angle in the horizontal plane projection is called the roll angle [9].When the ship's stability is discussed, rolling motion occupies an important position in the stability calculation [10], [11]. So when the difference between two models is compared, therefore, the research is done from the rolling motion in the paper.

In theory, when the rolling motion occurs, the roll angle of the side is the same. So the point A at central section side is selected from the whole bulk carrier segment in the rigid body model as a measurement. Point A on model 1 along the $\mathrm{x}$ axis which means the lateral deviation from the origin of mobile distance changing with time curve is shown in Fig. 6.

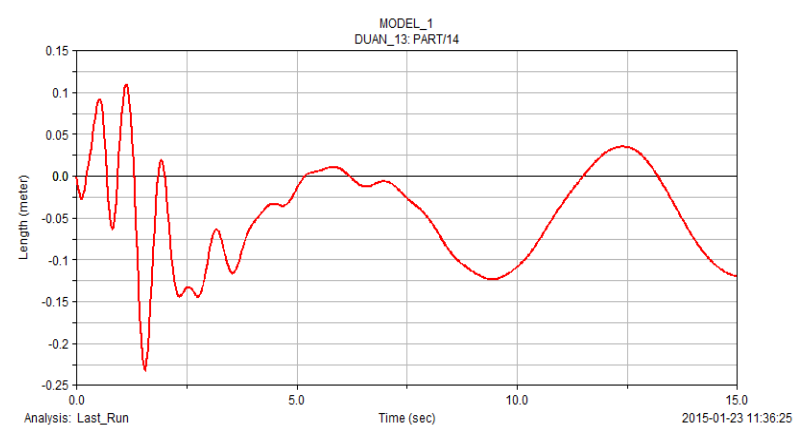

Figure 6. The lateral deviation curve of model 1.

It can be seen from the figure 6 that the maximum offset distance is $0.1249 \mathrm{~m}$ and the distance between the two maximum displacement points of the reciprocating swing is $0.1421 \mathrm{~m}$. The curve of the roll angle changes with time is shown in Fig. 7.

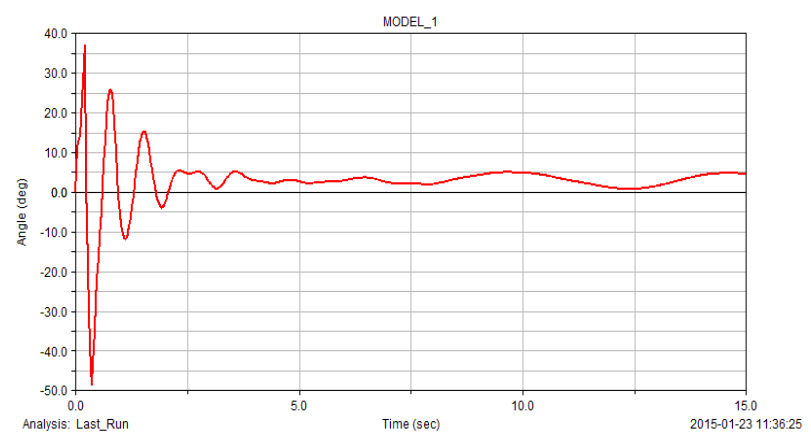

Figure 7. The roll angle curve of model 1.

It can be seen from the Fig. 7 that maximum roll angle is $5.101^{\circ}$ and the roll angle which the stability calculation of the original ship provides is $11.805^{\circ}$. model 1 cannot accurately simulate the actual status of the ship motion. If this model is used, the result comes out to be dangerous. 
Point A on model 2 along the $\mathrm{x}$ axis deviating from the origin's distance changing with time curve is shown in Fig. 8.

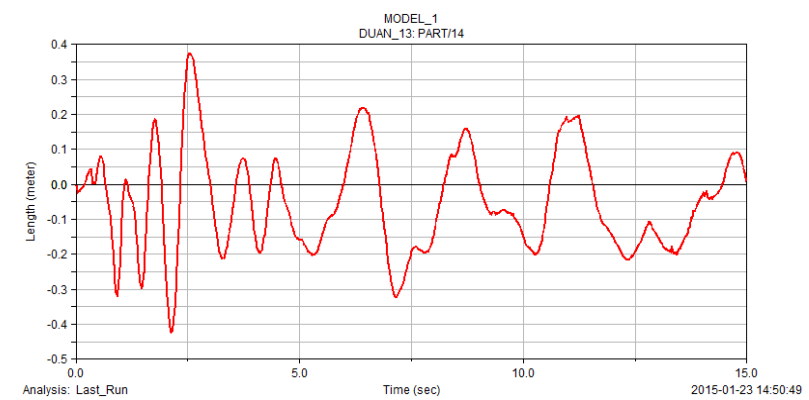

Figure 8. The lateral deviation curve of model 2.

It can be seen from the figure 8 that maximum offset distance is $0.3208 \mathrm{~m}$ and the distance between the two maximum displacement points of the reciprocating swing is $0.5443 \mathrm{~m}$. The curve of the roll angle changes with time is shown in Fig. 9.

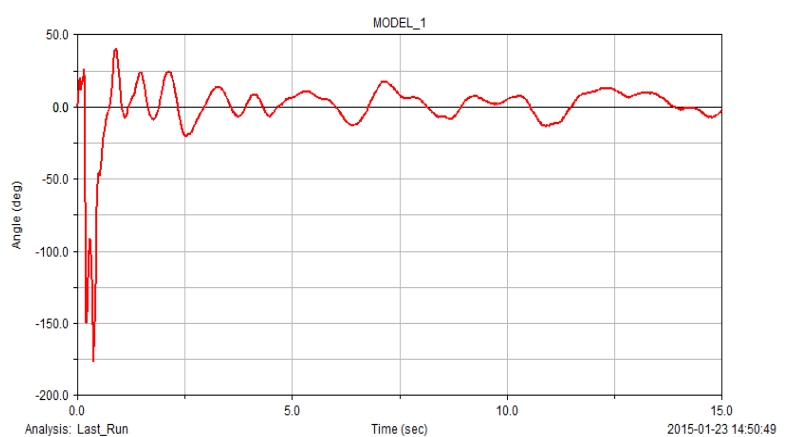

Figure 9. The roll angle curve of model 2.

It can be seen from the Fig. 9 that maximum roll angle is $17.6866^{\circ}$. It can be seen that in the case of no ballast water, the ship's maximum roll angle is greater than the largest stability calculation data are given. If this model is used, the result comes to be conservative.

Add ballast water on the model 2, lateral displacement changing with time is shown in Fig. 10.

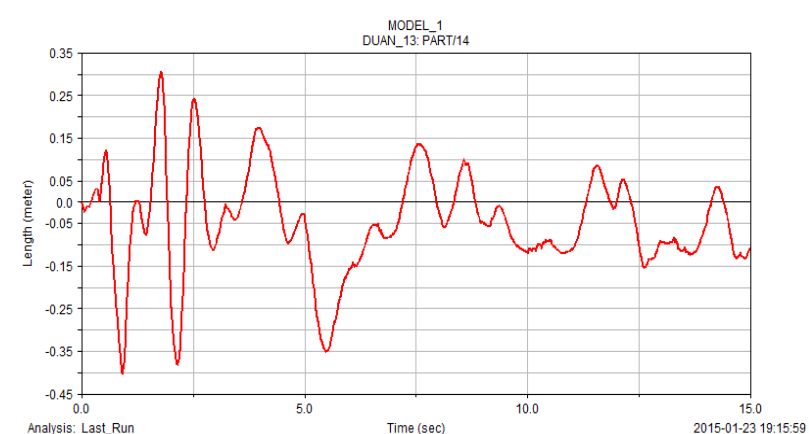

Figure 10. The lateral deviation curve with ballast water

It can be seen from the figure 10 that when at not stable state, the maximum distance is $0.4012 \mathrm{~m}$ and decreased by $0.0330 \mathrm{~m}$ compared to the distance of the displacement of the model without ballast water. Reduction is $7.60 \%$ of the model without ballast water. After reaching a stable state, the maximum distance is $0.1379 \mathrm{~m}$ and reduced by $57.01 \%$ compared with the model without ballast water. The maximum displacement distance is $0.2906 \mathrm{~m}$ when in reciprocating swing state and reduced by $46.61 \%$ compared with the model without ballast water.

Add ballast water on the model 2, roll angle changing with time is shown in Fig. 11.

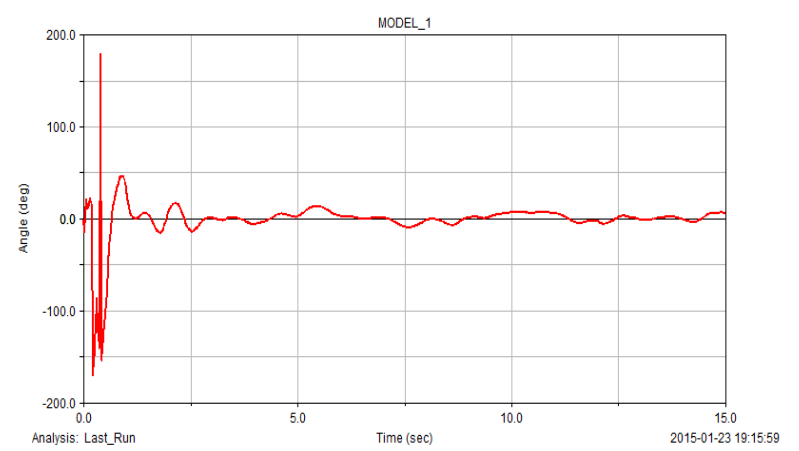

Figure 11. The roll angle curve with ballast water

It can be seen from the Fig. 11 that when at stable state, the maximum roll angel is $8.2667^{\circ}$ and decreased by $53.26 \%$ compared to the maximum roll angle of the model without ballast water. And the results meet the specification requirements.

\section{Conclusions}

The equivalent method of the relative movement goods on board is discussed in details. The ADAMS dynamic model is established based on moving trajectory of gravity-center for goods and to take rigid body geometric model with the trajectory as constraints. The difference of simulation methods for the different goods in carrier rolling is compared. The interact of relative moving objects with bulk carrier is discussed by using the ADAMS model. It is verified that the ballast water can maintain the ship's stability by means of the ADAMS model.

\section{References}

1. M.Z. Qiu, Stability and Navigating Safety of a ship in Following (Harbin Engineering University, 2008)(In Chinese)

2. H.S.Yang, Re-understanding of ship stability, Marine Technology 2,21-22(2003) (In Chinese)

3. L.B.Zhou, Ship in big storm of how to prevent the movement of goods, Ship maneuvering in big waves 1, ,2006) (In Chinese)

4. J.H .Cai, Discussion on the movement of ship cargo, Marine Technology1, 33-34 (2012) (In Chinese)

5. R.Wang, Study of Anti-heeling System Design for Ship(Harbin Engineering University,2007)(In Chinese)

6. Z.W. Chen, Y.L. Dong, Multi body dynamics simulation based on MSC Adams(China Water Conservancy and Hydropower Press,BeiJing,2012) (In Chinese)

7. Z.M. Wan, F.Wu, H. Li, P. Zhu, Q.T.Xu, R.G. Zhang, Study on the grain storage under different conditions, Grain Storage41 (5) ,10-15(2012) (In Chinese) 
8. X.L.Zhou, H.Z.Li, S.Q.Yu, Study on static angle of natural sediment accumulation (2014 Conference on Computational Mechanics of granular materials,2014) (In Chinese)

9. P.Y. Li, T.C. Feng, Y.M.Qiu, The motion of the three body ship, Chinese shipbuilding44(01), 24-30 (2003) (In Chinese)
10. S.G.Zhang, Theoretical study on roll stability of ships in large waves, Chinese Journal Of Ship Research1(01), 50-53 (2006) (In Chinese)

11. Y.K. Hu, Study on the large rolling motion and stability of the ship in waves(Harbin Engineering University, 2011) (In Chinese) 